\title{
Technology in the Hands of Managers: Email Use by Australian and Hong Kong Managers
}

\author{
Paul L. Nesbit \\ Macquarie University \\ E-Mail: paul.nesbit@mq.edu.au \\ Suzan Burton \\ Macquarie University \\ E-Mail: suzan.burton@mq.edu.au
}

\begin{abstract}
Since communication plays an important role in the work of managers they are likely to be significant users of e-mail in their daily work. Managers are also influential in technology acceptance and in policy formation. Thus investigating e-mail use by managers is particularly relevant to advance the understanding of organisational efforts to maximize benefits and to minimize problems with e-mail communication. This paper reports on the findings of a survey of managerial use of e-mail within Australian and Hong Kong. The results suggest that while the primary use of e-mail is currently for inter-organisational communication, e-mail is consuming large amounts of managerial time, particularly for more senior managers. A significant proportion of e-mail received did not appear to contribute to productivity, with both personal e-mails and marketing emails constituting a significant proportion of received e-mails. The study suggests that email use will grow as individual and organisational experience increases. We suggest that managers need to consider the time demands caused by the growing use of e-mail.
\end{abstract}

Keywords: E-Mail, Managerial Work Patterns, Policy, Work Time 


\section{INTRODUCTION}

Since the mid 1990s, the Internet has had a dramatic impact on the amount and speed of organisational communication. In 2001 more than half of all workers in one US study used a computer at work, and the most common use of a computer was reported to be for connecting to the Internet or accessing e-mail (Hipple \& Kosanovich, 2003). Approximately $98 \%$ of workers who have Internet access at work use e-mail at their site of employment (Fallows, 2002). E-mail clearly presents advantages for organisations, allowing message and file transmission to anyone connected to the Internet anywhere in the world, at any time. The impact of e-mail will vary between and within organisations, but researchers have agreed that e-mail has significantly changed life in the organisation (Minsky \& Morin, 1999).

While e-mail communication has obvious benefits for businesses, the disadvantages of the medium are also becoming apparent. Popular reporting of e-mail use in organisations often highlights its negative aspects, such as communication and information overload and the excessive time spent dealing with e-mail (Harmon, 2001). The ease of message composition and transmission to multiple recipients at the push of a button can result in excessive numbers of e-mails for organisations and individuals. It has been estimated that managers can spend two to three hours each day reading, sending, and responding to e-mails (Hymowitz, 2000). Since e-mail is playing an increasingly significant role within organisational communication, organisational leaders may wish to give more attention to enhancing the efficiency and effectiveness of its use.

\section{MANAGERIAL EMAIL USE IN ORGANISATIONS}

Investigating e-mail use by managers is particularly relevant to advance the understanding of organisational efforts to maximize benefits and to minimize problems with e-mail. Since communication plays an important part in the role of managers they are likely to be significant users of e-mail in their daily work. Managers are also influential in technology acceptance and in policy formation. The use of e-mail by a group leader has been found to be positively related to e-mail usage by the rest of the group (Minsky \& Marin, 1999; Romm \& Pilskin, 1999). Individuals' knowledge of, and experience with, e-mail has been shown to influence their perceptions of the medium (Carlson \& Zmud, 1999). If managers perceive problems with their experiences, they are more likely to initiate corrective action such as policy guidelines and training. Most importantly, senior management time is a scarce and expensive resource for 
organisations, and it is important for organisations to understand the impact of e-mail on managerial time allocation, and optimize managerial use of e-mail.

Despite the importance of managers as a group of users of e-mail, there are no systematic studies of their usage patterns and influences on that use. Sullivan's (1995) study of e-mail use in a government setting focused on the amount of time spent dealing with e-mail by secretarial, professional and senior staff use but did not specifically examine managerial experiences. Stevens, Williams and Smith (2000) examined managerial usage of the Internet but the sample was only small (some $18 \%$ of 30 respondents) and so general conclusions could not be drawn. One cross-cultural study of media choice found that managers on average sent 23 e-mails per week and received some 46 messages per week (Rice, D’AMbra \& More, 1998). However, their study was focusing on media richness rather than understanding and advancing knowledge of the influences of managerial e-mail use. More recent surveys of work related e-mail use have shown a substantial increase in its use of e-mail but the whether this also applies to managers is unknown. Fallows (2002) for example, found that $60 \%$ of those who use email at work receive 10 or fewer messages on an average day, while $23 \%$ of respondents receive more than 20 e-mails in a day. The survey also showed that $78 \%$ of work emailers sent 10 or fewer messages on a typical day and only $11 \%$ sent more than 20 emails. Unfortunately, specific data on the role of the e-mail users was not collected and so specific managerial use remains unknown. Given the evolving nature of e-mail use in organisations (Van der Hooff, 2005) and the importance of managers as a user group, there is a clear need to explore and benchmark their e-mail use patterns as well as understand the influences on that use and evaluations of their experiences.

Communication theory suggests that choice of a communication medium will be influenced by both individual characteristics and by social influence, such as group norms, organisational structure and social learning in the workplace (Minsky \& Marin, 1999). Early studies of e-mail use in the US and Canada showed that younger people and males tend to be higher users of the Internet and e-mail due to both gender and socioeconomic factors (Bimber, 2000). More recent reports, however, show little difference in the frequency of Internet use by men and women, but continue to show variation in Internet use according to age (Hipple \& Kosanovich, 2003). The seniority of the manager may also influence e-mail use due to senior managers' central role in organisational communication channels. Finally, a manager's length of experience using e-mail is likely to influence use, since familiarity with computers, e-mail software and its 
benefits are likely to encourage continued use.

In addition to managerial characteristics a number of organisational characteristics - organisation size and length of experience of e-mail use are likely to influence e-mail usage patterns. Large organisations are likely to be geographically dispersed, thus increasing the likelihood of use of electronic forms of communication. Even if organisations are located in one area, large staff numbers are likely to make email an attractive form of communication (Huber, 1990). Organisational length of use is also likely to encourage a culture of e-mail communication, as well as increase individual managers' experience and familiarity with the medium (Van der Hooff, 2005) suggesting a positive relationship between organisational size and experience with e-mail and managerial use of e-mail. Thus individual managerial characteristics of age, gender, seniority and length of use, as well as organisational characteristics of size and length of e-mail are suggested to be associated with increased managerial e-mail use.

\section{CULTURAL VALUES AND EMAIL USE}

Given the increasing internationalization of business and the evidence of growing reliance on e-mail, the question of whether differences exist in the managerial use of email between different countries may have important coordination and communication implications (Lee, 2000). National culture plays an important role in influencing thoughts and action and has been shown to have an impact on the use of information technologies (Calhoun, Teng, \& Cheon, 2002; Rice, D’Ambra, \& More, 1998). For example, Straub (1994) argued that facsimile plays a more important role in Japanese business communication than e-mail because facsimile provides more flexibility in using the Japanese character set. Huang, Ming-Te, and Bo (2003) studied the relationship between power distance and e-mail acceptance and noted that e-mail has an equalizing effect which may run counter to perceived usefulness in cultures with high power distance and so limit its acceptance.

\section{METHODOLOGY}

One hundred and sixty eight managers (88 in Australia and 80 in Hong Kong) completed a questionnaire concerning their use of e-mail at work for both business and private use. All were practicing managers engaged in part-time management study. Questionnaires were provided to participants of a number of post-graduate management education courses and gathered in the following class held a week later. Participation was 
voluntary and anonymous. The response rate varied between classes but was relatively high at 55\% for the Australian participants and 43\% for the Hong Kong Participants. Given the anonymous nature of the survey we were not able to identify any underlying bias between those who responded and those who did not. Respondents were asked to record the number and type of e-mails received (eg. "I received e-mails over the course of 24 hours", "Out of all my e-mails, are of little interest to me", "I sent e-mails over the course of 24 hours"), and sent over one 24 hour period, and to estimate the time spent on e-mail over that day. The survey also investigated organisational characteristics (such as size, length of use of e-mail) and personal characteristics of the respondent (such as age, gender, and length of use of e-mail), which were proposed to be associated with use of e-mail. Seniority of the manager was also recorded.

Self-reporting of e-mail type is likely to produce a bias towards under-reporting of personal use of e-mails. Respondents may be reluctant to report very high personal use of e-mail during work time. Similarly, e-mails with mixed social and business content are more likely to be reported as primarily business in content. Without obtaining a written record of an individual's e-mail output and independent coding of e-mails, it would be difficult to obtain a more objective measure of the extent of personal e-mail use, and this method would be unacceptable in most circumstances due to privacy constraints. It was therefore decided to accept self-reporting of e-mail type, with the understanding that there was likely to be a bias in favor of under-reporting personal use of e-mails. Data was cleaned to check for data entry errors, and analyzed using t-tests, ANOVA, univariate, multivariate and logistic regression and chi-square tests to reflect the different types of data and statistical tests required.

Australian and Hong Kong cultures are seen to vary on power distance and individualism-collectivism dimensions, two cultural dimensions identified by Hofstede (1991) whose cultural model has underpinned much of the academic research on culture (Calhoun, Teng, \& Cheon, 2002). Thus our sample allows insights into the degree that email-use may be influenced by two distinctly different cultures. Australian and Hong Kong comparisons also control for technological factors in e-mail use. At the time of the survey Australia and Hong Kong had high internet penetration among the general population and within business (Minges, 2003, ABS, 2001; Hong Kong Census and Statistics Department, 2002).

In the following section we report on the results from the two surveys that provides 
insight into the email usage patterns of managers and the influences on that pattern. Additionally, we examine for differences between Australian and Hong Kong managers in order to explore the potential for cultural effects on email use.

\section{RESULTS}

Gender of the respondents was roughly equal; (51.5\% male, 48.5\% female). Other respondent characteristics are shown in Table 1. There were no significant differences between the Australian and Hong Kong samples in terms of age, seniority, length of personal or organisational use of e-mail, or organisational size $(p>0.10)$. Australian respondents were more likely to be male $(\mathrm{p}=0.001)$, reflecting a higher proportion of males in the sampling frame.

Table 1 Respondent statistics

\begin{tabular}{lrr}
\hline & Mean & \multicolumn{1}{c}{ Std } \\
\hline Age & 34.0 & 6.1 \\
Levels from CEO & 3.6 & 1.9 \\
Personal use of e-mail (yrs) & 5.6 & 3.1 \\
Organisation size (staff no) & 957.1 & 49.6 \\
Organisational use of e-mail (yrs) & 5.9 & 3.3 \\
\hline
\end{tabular}

\section{E-Mail Usage Patterns}

Managers typically used more than one e-mail address, with the modal number of email addresses being two (53\% of respondents). The work address was nearly always the most commonly used address (86.3\% of respondents). Respondents estimated the amount of time that they spent on e-mail during the 24-hour period under study, with details shown in Table 2 . The modal category, estimated by $28.6 \%$ of respondents, was more than two hours spent on reading and/or answering e-mail. Clearly, e-mail is consuming a significant portion of work time. Not surprisingly, people who send a lot of e-mail tend to receive a lot of e-mail $(\mathrm{r}=0.727, \mathrm{p}<0.001)$. 
Table 2 Estimated time spent on e-mail per day

\begin{tabular}{lcc}
\hline & & Cumulative \% \\
\hline 0-5 minutes & 0 & 0 \\
5-30 minutes & 21.9 & 21.9 \\
30 minutes -1 hour & 25.7 & 47.6 \\
1-2 hours & 23.8 & 71.4 \\
More than 2 hours & 28.6 & 100 \\
\hline
\end{tabular}

Table 3 shows the mean frequency and type of daily e-mails received at work by the sample. There were no significant differences between Australian and Hong Kong samples in the numbers of e-mails sent or received, in the frequency of social or direct selling e-mails received, the percentage of e-mails that were received from within the organisation ( $\%$ inter-org received), or the percentage of e-mails which were seen to be of little interest to the recipient. The percentage of e-mails sent outside the organisation and the percentage sent to only one person (\% unique sent) were significantly different between the two countries $(p=0.05)$, however these results may be due to chance, due to the number of tests performed. At a Bonferroni adjusted level of significance ( $p=$ $0.05 / 11=0.005)$ the difference in e-mails sent outside the organisation $(p=0.009)$ just failed to reach significance and there were no other significant differences between the countries on any of the variables in Table 3.

Table 3 Descriptive statistics

\begin{tabular}{lllllc}
\hline \multicolumn{1}{c}{ Mean } & Australia & Hong Kong & All & $\begin{array}{c}\text { All } \\
\text { (std dev) }\end{array}$ & $p$ \\
\hline Received & & & & & \\
No. received total & 29.0 & 24.6 & 26.9 & 22.40 & 0.208 \\
\% unique received & 46.7 & 45.0 & 46.1 & 11.90 & 0.184 \\
\% inter-org received & 61.8 & 64.4 & 63.2 & 18.00 & 0.465 \\
\% social received & 14.6 & 15.1 & 14.9 & 4.90 & 0.505 \\
\% replied to: & 45.7 & 53.1 & 49.1 & 13.10 & 0.930 \\
\% of little interest & 22.8 & 20.2 & 21.6 & 7.90 & 0.183 \\
\% selling & 8.9 & 7.1 & 8.2 & 6.70 & 0.414 \\
\hline
\end{tabular}


Table 3 Descriptive statistics (Continued)

\begin{tabular}{llllll}
\hline \multicolumn{1}{c}{ Mean } & Australia & Hong Kong & All & $\begin{array}{c}\text { All } \\
(\text { std dev })\end{array}$ & $p$ \\
\hline Sent & & & & & \\
No. sent total & 14.2 & 12.3 & 13.3 & 13.30 & 0.287 \\
\%. sent outside & 38.4 & 28.4 & 33.8 & 4.80 & 0.009 \\
\% unique sent & 49.0 & 38.1 & 44.4 & 6.20 & 0.020 \\
\% social sent & 18.0 & 16.2 & 17.3 & 3.20 & 0.257 \\
\hline
\end{tabular}

Table 3 also provides details on the level of use of e-mail for primarily social reasons, and perceptions of the relevancy of e-mails received. Reported social use of emails is $14.6 \%$ for messages received by Australian managers and 15.1\% for Hong Kong managers, however the difference was not significant $(p=0.505)$. Reported frequency of use of e-mail for primarily social reasons was significantly and negatively associated with seniority in the organisation, with more senior managers reporting a lower proportion of social e-mails $(p=0.006)$.

\section{Formal Email Policy}

The survey also asked about the existence of organisational e-mail policies. Australian organisations were more likely to have a formal e-mail policy $(63.6 \%)$ than Hong Kong organisations (33.4\%). This difference in the existence of policies concerning e-mail use was significant $(p=0.002)$. The difference was not explained by any difference in the size of organisations sampled, since the difference was significant, even after allowing for the size of the organisations sampled in each country $(p=0.001)$.

Organisational e-mail policies appear to be of two major types; those which were designed to protect the organisation from legal risk (e.g. barring pornographic content or material that could be associated with harassing others) and policies which attempted to limit e-mail proliferation (e.g. suggesting limits on the number of copies or attachments). Very few policies mentioned personal use of e-mail, and where policies did discuss personal use of e-mail, such use was permitted as long as it did not distract from work and otherwise conformed with organisational policies regarding content suitability. While the study did not test whether e-mail policies were effective in restricting transmission of 
unsuitable content, there was no evidence that having a policy limited proliferation of inappropriate e-mails. In fact, the data were in the opposite direction, with employees from organisations with an e-mail policy reporting higher proportions of e-mails which were of little or no interest to them (means $25.5 \%, 21.1 \%$ ) though the difference was not significant $(p=0.428)$. Existence of a policy was not associated with any difference in the proportion of social e-mails $(p=0.745)$.

\section{Predicting the Number of Emails Received}

The absence of statistically different patterns of usage between Australian and Hong Kong managers suggests that despite the cultural differences between the two countries, managerial e-mail usage is directly comparable. As a result, the sample was combined to model the factors that predict e-mail usage. Table 4 displays the results of univariate and multivariate analysis to predict the number of e-mails received.

Table 4 Predicting number of e-mails received: multivariate analysis

\begin{tabular}{lllll}
\hline IV & $\beta$ & St.Dev & T & $\mathrm{p}$ \\
\hline Length of use (individual) & 4.789 & 1.401 & 3.42 & 0.001 \\
Length of use (organisational) & 3.146 & 1.347 & 2.34 & 0.021 \\
Levels from CEO ${ }^{\mathrm{a}}$ & -2.263 & 1.124 & -2.01 & 0.046 \\
Organisation size & 1.857 & 1.095 & 1.70 & 0.092 \\
Age & -1.684 & 1.550 & -1.09 & 0.279 \\
\hline$R^{2}=0.255$ : Adjusted $R^{2}=0.226^{\text {a }}$ Coded CEO $=$ level 1, next highest level = 2 etc
\end{tabular}

\section{DISCUSSION}

The results support anecdotal reports that e-mail is consuming large amounts of managerial time. On average, Hong Kong and Australian managers received 26.9 e-mails a day at work, and sent about 13.3 e-mails. This compares to US reports that the average corporate user receives about 30 messages a day (Hymowitz, 2000). As would be expected, respondents who sent a lot of e-mail tended to receive a lot of e-mail $(p<0.001)$. Thus e-mail may generate an increasing spiral of use - sending more e-mail will typically generate more responses to which the sender replies, creating new demands for messages and replies. This view was supported by the results which showed that people who had used e-mail for longer were significantly more likely to receive larger numbers of e-mails $(p<0.001)$ and that a high percentage $(53.3 \%)$ of received e-mails 
were copies of messages sent to other people.

While an early study of e-mail by Sullivan (1995) found that e-mail use was more prevalent at lower levels of the organisational hierarchy, we found that higher use of email is related to seniority of level in the organisation, even allowing for factors such as personal or organisational experience with e-mail. The manager, being at the centre of the business communication network, appears to be receiving more e-mails, as e-mail becomes an entrenched component of organisational communication. Thus this changing pattern of use of e-mail is likely to result in a large increase in time demands for senior management.

The average manager in the survey estimated that they were spending one to two hours on e-mail each day. About two-thirds (63.2\%) of the e-mails received originated from inside the managers' organisations, indicating the dominant use of e-mail is for intra-organisational communications. However, $21.6 \%$ of e-mail was said to be of little interest to the recipient suggesting that much of the time spent on e-mail may not be useful. It is particularly worrying that senior managers were significantly more likely to receive increased numbers of e-mail messages than their junior and middle level colleagues. Thus, the increasing use of e-mail in organisations, many of which have little interest for recipients, suggest a large and growing problem with unwanted e-mail communication, with the problem greatest for managers at a higher level within the organisations.

A potential problem for organisations is the extent to which e-mail can be used for non-business purposes. In this study, almost one in six e-mail messages (14.9\%), was classified as primarily social. Moreover, as discussed above, there are reasons to suggest that any self-report of the percentage of personal use of e-mail will be an under-estimate, so this figure may be a conservative estimate of personal e-mail use. In the sample organisations, $55.9 \%$ had instituted specific policies concerning e-mail usage, but there was no evidence to suggest that presence of an e-mail policy was associated with lower numbers of e-mails. E-mail policies to date appear to have largely followed the pattern of telephone usage within the organisation; at middle management level and above, personal use is generally permitted under the assumption that use does not detract from work performance. However if use of e-mail continues to rise, this practice may be questioned, because the nature of e-mail usage appears to have the potential to disrupt work more than telephone calls, because of the easy ability to send a message to multiple people at the same time. It is interesting to consider why this potential major drain on staff time is 
not addressed in most e-mail policies. While many organisations are vigilant to the need to monitor for pornographic content and have established filters for this type content, it is more difficult to assess social content of e-mails. Thus, in the absence of data, organisations may not recognize the extent of e-mail use for primarily social purposes, and hence do not see it as a problem.

It is also possible that organisational leaders recognize that even for social e-mail there are intangible benefits that accrue to the organisation, such as fostering communication, and/or replacing other methods of informal communication. This is supported by some reports that suggest that e-mail is partly substituting for other forms of communication, such as the telephone (Dimmick, Kline, \& Stafford, 2000). Nevertheless, organisations will increasingly need to consider the extent to which e-mail is being used for personal use, and the drain that this is creating on staff time and seek ways of controlling e-mail use without being seen as instituting draconian policies.

Despite similar levels of actual e-mail use between the two countries, Australian organisations were significantly more likely to have instituted formal e-mail policies. This finding is consistent with previous studies that suggest that Eastern organisations rely less on formal structuring of employee behavior, through explicit rules and policies, than Western organisations (Pugh and Hickson, 1976, Redding, 1980; Whitley, 1991). As expected, existence of an e-mail policy was positively correlated with larger numbers of staff $(p<0.001)$. There was also support for the proposition that organisations with longer experience with e-mail were more likely to have an e-mail policy $(p=0.017)$. It is possible that longer experience with e-mails and the greater number of e-mails which this generates reveals problems that management attempts to address with a policy. However, there was no evidence that existence of a policy was associated with lower use of e-mails, or with a lower percentage of irrelevant e-mails. In fact, the data showed a weak trend existed in the opposite direction.

In addition to formal e-mail policies at the organisational level, limited inter-country differences were also evident. Australian managers were more likely to send e-mails outside the organisation and to send e-mails to individual recipients than Hong Kong managers (see Table 3). These differences in e-mail use may reflect cultural influences evident in more traditional forms of social communication (Lee, 2000). Australian managers, like other Western managers, tend to value individualism and emphasise personal initiative in communication (Hofstede, 1991). In contrast, Hong Kong managerial behaviour is heavily influenced by Confucian values and greater power 
distance, which are exhibited by deference and respect to those at higher levels of the hierarchy of the organisation (Huber, 1990), thus Hong Kong managers may see such external communication as inherently part of the responsibility of senior managers. Similarly the greater tendency of Hong Kong managers to send e-mail to more than one person may also reflect the desire for harmony and to show respect for colleagues. Australian managers might tend to send e-mail to others only if it serves some task related purpose. While these views are speculative, they are consistent with other crosscultural research (Lee, 2000; Huang, et.al. 2003), and suggest that future research could profitably focus on the cultural nuances of managerial e-mail use.

Perhaps one of the most important implications that can be drawn from the data is that the underlying pattern of managerial work is undergoing a fundamental transformation. This transformation arises not only from the fact that contemporary managers spend a substantial amount of their time composing, reviewing, and responding to e-mails but also from the fact that these activities are typically carried out in one's office, isolated from others. The average manager in our study spent more than one hour per day in dealing with e-mails, with nearly $30 \%$ of managers spending more than two hours per day. This represents a large amount of a manager's day spent involved in a relatively isolated activity, which is in sharp contrast to the results of Kotter's (1982) study of managerial work patterns, which showed that managers tended to spend most of their time engaged with others in face-to-face communications, a pattern often characterized by spontaneous interactions. Few general managers in Kotter's study spent more than $30 \%$ of their work time on solitary activities, with some spending as little as $10 \%$ of their time alone. Thus, the growing use of e-mail may be fundamentally changing the nature of managerial work by discouraging personal interactions. The pattern of spontaneous corridor interactions, observed by Kotter in the 1980's may be being replaced by the manager who sits alone in the office, responding to messages, skilled in computer based interactions but with only limited interpersonal communication skills.

\section{MANAGERIAL IMPLICATIONS}

While e-mail has the potential to be less disrupting because it does not require immediate attention, recent research has shown that most users opened messages, on average, one minute and 44 seconds after arrival, followed by a period of interruption before the recipient returned to their pre-e-mail activity at the former rate (Jackson, Dawson, \& Wilson, 2003). Given the potential for disruption, organisational leaders may 
wish to give more attention to enhancing the efficiency and effectiveness of e-mails. One possible avenue is to develop policies on such issues as the appropriateness of e-mails, length of e-mails and attachments, and provide protocols and suggested strategies for distribution and reply. Training for clarity of e-mails, and in recognising the limitations of e-mail as a form of communication, may also help to reduce excessive and irrelevant e-mails. Other usage strategies include educating employees about the negative effect of interrupts (Solingen, Berghout, \& Latum, 1998), decreasing the frequency of automatic email checking, and changing the method of e-mail notification (Jackson, Dawson, \& Wilson, 2003).

\section{FUTURE RESEACH}

While this research did not investigate the extent to which the respondents' organisations were using filtering software to detect and delete junk e-mail messages, the results suggest that there will be increasing demand for this software as the number of junk e-mails increases. Such software is likely to be only a partial solution to the time demands created by e-mail, however, since this study suggests that a large number of emails which are seen to be time-wasting are from within the organisation, and are unlikely to be filtered by such software.

While the data reported here reflects the e-mail usage of a relatively small sample of managers in Australia and in Hong Kong, it nevertheless provides a useful benchmark of contemporary managers' use of e-mail in these two countries. These data provide a basis for investigating evolving patterns of managerial e-mail use. While we found few strong differences in e-mail usage by Australian and Hong Kong managers, differences may emerge as a by-product of different approaches to policing e-mail in organisations. This study did not collect data on respondents' industry type, and it is possible that there are systematic differences in e-mail use in different industry sectors. Another useful direction for further research into e-mail use in organisations would be to explore the potential transformative impact of e-mail on the managerial role and interpersonal communications within the organisation.

\section{REFERENCES}

ABS. (2001). Yearbook Australia 2002: Communication and Information Technology.: Australian Bureau of Statistics.

Bimber, B. (2000). Measuring the gender gap on the Internet. Social Science Quarterly, 
$81(3), 868-876$.

Calhoun, K.J., Teng, J.T., \& Cheon, M.J. (2002) Impact of national culture on information technology usage behavior: AN exploratory study of decision making in Korea and the USA, Behavior and Information Technology, 21 (4), 293-302

Carlson, J. R., \& Zmud, R. W. (1999). Channel expansion theory and the experiential nature of media richness perceptions. Academy of Management Journal, 42 (2), 153170.

Dimmick, J., Kline, S., \& Stafford, L. (2000). The gratification niches of personal e-mail and the telephone: Competition, displacement, and complementarity. Communication Research, 27 (2), 227-248.

Fallows, D. (2002). E-mail at Work. Pew Internet and American Life Project, 1-26.

Harmon, A. (2001, Dec.24). You've got mail. Lots of it, and it's mainly junk. The New York Times. 18

Hipple, S., \& Kosanovich, K. (2003). Computer and Internet use at work in 2001. Monthly Labor Review, Washington, 126 (2), 26-35.

Hofstede, G. (1991). Cultures and organisations: software of the mind. London: McGraw-Hill.

Hong Kong Census and Statistics Department (2002), Annual Survey on Information Technology Usage and Penetration in the Business Sector (2002).

Huang, L, Ming-Te, L., \& Bo, W.K (2003). The impact of power distance on e-mail acceptance: Evidence from the PRC, Journal of Computer Information Systems, Fall, 93-101.

Huber, G. P. (1990). A theory of the effects of advanced information technologies on organisational design, intelligence, and decision making. The Academy of Management Review, 15 (1), 47-71.

Hymowitz, C. (2000, Sep 26). Flooded with e-mail? Try screening, sorting, or maybe just phoning, pp. B1.

Jackson, T., Dawson, R., \& Wilson, D. (2003). Reducing the effect of e-mail interruptions on employees. International Journal of Information Management, 23 (1), 55-65.

Kotter, J. P. (1982). What effective General Managers really do. Harvard Business Review, 60 (6), 156-167.

Lee, O. (2000). The role of cultural protocol in media choice in a Confucian virtual workplace. IEEE Transactions on Professional Communication, 43 (2), 196-200. 
Minsky, B. D., \& Marin, D. B. (1999). Why faculty members use e-mail: The role of individual differences in channel choice. The Journal of Business Communication, 36 (2), 194-217.

Minges, M (2003) Broadband as a commodity: Hong Kong, China Internet Case Study, International Telecommunication Union, May 2003.

Pugh, D. S., \& Hickson, D. J. (1976). Organisational Structure in its Context. London: Saxon House.

Redding, S. G. (1980). Cognition as an aspect of culture and its relation to management process: an exploratory review of the Chinese case. Journal of Management Studies, $12(2), 127-148$.

Rice, R. E., D'Ambra, J., \& More, E. (1998). Cross-cultural comparison of organisational media evaluation and choice. Journal of Communication, 48 (3), 3-25.

Romm, C., \& Pliskin, N. (1999). The role of charismatic leadership in diffusion and implementation of e-mail. The Journal of Management Development, 18 (3), 273290.

Solingen, R., Berghout, E., \& Latum, F. (1998). Interrupts: Just a minute never is. IEEE Software, 15, 97-103.

Stevens, P. M., Williams, K. P., \& Smith, M. C. (2000). Organisational communication and information processes in an Internet-enabled environment. Psychology \& Marketing, 17 (7), 607-632.

Straub, D. (1994). The effect of culture on IT diffusion: e-mail and fax in Japan and the US. Information Systems Research, 5, 23-36.

Sullivan, C. B. (1995). Preferences for electronic mail in organisational communication tasks. The Journal of Business Communication, 32 (1), 49-63.

Van der Hooff (2005). A learning process in e-mail use - a longitudinal case study of the interaction between organisation and technology, Behaviour \& Information Technology24 (2), 131-145.

Whitley, R. D. (1991). The social construction of business systems in East Asia. Organisation Studies, 12 (1), 1-28. 
Contemporary Management Research 212 